\title{
LA DEMANDA DE ELECTRICIDAD EN LA REGIÓN CENTRAL DEL PERÚ: 2001- 2010*
}

\author{
Villaverde Montoya, Oscar ${ }^{1}$ y Campos Arias, Miguel² \\ Facultad de Economía de la Universidad Nacional del Centro del Perú1 - Banco Central de Reserva del Perú \\ -Sucursal Huancayo, Perú2.
}

\begin{abstract}
RESUMEN
La planificación del sector eléctrico es de suma importancia para el crecimiento de una economía y, de manera más precisa, la planificación de la producción eléctrica de Electrocentro S.A. es compatible con en el crecimiento sostenible de la región central del país. La eficiencia en la gestión de la empresa requiere de estimaciones precisas de la demanda eléctrica, a fin de evitar excesos en la producción que originaría sobrecostos y pérdida de beneficios. En el trabajo de investigación se estima la demanda de electricidad de la región central del país, a fin de encontrar los factores que determinan el comportamiento de la demanda, así como la proyección global de la demanda eléctrica. En la estimación de la demanda global se ha utilizado la metodología de Panel de Datos y para la proyección de la demanda eléctrica se ha recurrido a los modelos Arima.
\end{abstract}

Palabras clave: Demanda de electricidad, estimaciones y proyecciones.

\section{DEMAND OF ELECTRICITY IN THE CENTRAL REGION OF PERU: 2001-2010}

\begin{abstract}
The planification in the electrical sector is of extreme importance for the growth of the economy and, most precisely the planification of the electric production of ELECTROCENTRO S.A. company is compatible with a sustainable growth in the Central Región of the country the efficiency in the performance of the company requires of precise estimations of the electrical demand as like the factors that determine it in order to avoid excesses in the production that would cause over costs and the loss of profits in the present work of investigation the demand of electricity in the Central Región of the country, has been estimated en order to find the factors that determine the behaviour of the demand, as well as the global projection of their requirement. In that estimation of the global demand a methodology y of a panel of data has been used and the arima models for the projection of the electrical demand.
\end{abstract}

Key words: Demand of electricity, estimations and projection.

\footnotetext{
* Este trabajo de investigación fue recibido el 20/09/2007, retornado para su revisión el 15/01/2008 y aprobado para su publicación el 20/07/2008.

1. Email: villaverde.o@pucp.edu.pe.
} 


\section{INTRODUCCIÓN}

La planificación del sector eléctrico es de suma importancia para el crecimiento sostenible de las economías y, de manera más precisa, la estimación de demanda de electricidad constituye una de las herramientas fundamentales en la planificación del sector eléctrico tanto a nivel nacional, regional y local. Para Electrocentro S.A. la producción de la energía eléctrica requiere de estimaciones precisas de la demanda, debido a que si la producción es mayor que la demanda se genera sobrecostos y pérdidas para la empresa. Asimismo, para plasmar las nuevas inversiones de la empresa se requieren de proyecciones precisas de la demanda eléctrica a fin de garantizar la rentabilidad del proyecto.

El problema que enfrenta Electrocentro S.A. es que no cuenta con modelos para la estimación de la demanda eléctrica que sirva en la toma de decisiones de producción e inversión. En el trabajo se construye un modelo de demanda de electricidad a fin de determinar los factores que explican el comportamiento de la demanda de electricidad, así la proyección global de la demanda eléctrica, en la en la región centro del Perú.

En el estudio se estima y se proyecta la demanda de electricidad para clientes regulados de baja tensión y media tensión utilizando la metodología de panel de datos y modelos Arima. La demanda de electricidad en la zona de concesión e influencia de Electrocentro S.A. considera 334 distritos en el mercado regulado de BT y 115 distritos en el mercado regulado de MT.

Chumacero (1996) realiza un estudio de la demanda eléctrica para zona central de Chile, donde propone una especificación no lineal en la variable dependiente ingreso, de modo que se obtiene la elasticidad variable a lo largo del tiempo y no constante como en las especificaciones logarítmicas. La no linealidad de la estimación permite evaluar la hipótesis de saturación, según el cual la elasticidad ingreso tiende a reducirse conforme se incrementa el nivel de ingreso.

En el caso Argentina, Mateos, Rodríguez y Rossi (1999) elabora un modelo estructural de equilibrio del mercado eléctrico, donde se estima la oferta y la demanda de electricidad en un sistema de ecuaciones simultaneas con variables instrumentales. En este modelo la oferta depende de las condiciones hídricas de las cuencas relevantes para el sistema de generación y los precios de los combustibles que afectan el costo de la generación térmica.

Moral Caicedo, J. y Vicente Otero, J. (2003), plantean un modelo de demanda de Energía Eléctrica que contempla y captura efectos tanto de corto y largo plazo ellos inciden fuertemente en diferenciar estos efectos, “....La distinción entre corto y largo plazo es esencial en la explicación de la demanda de energía eléctrica ya que a corto plazo las variaciones de demanda suelen estar ligadas a la mayor o menor intensidad de uso del equipamiento existente (actividad económica, variables climáticas y condiciones de trabajo); sin embargo, a mediano y largo plazo, la combinación de variables económicas (renta, ahorro, tipos de interés, expectativas...), demográficas y tecnológicas (construcción de nuevas viviendas, acumulación y renovación del equipamiento doméstico, crecimiento de la superficie comercial, equipamiento productivo industrial, transporte, etc ) afectan a variables claves en la demanda de energía eléctrica.

En cuanto a la metodología de Panel de Datos destaca el trabajo de José Benavente y otros (2004), donde se estima la demanda residencial usando un panel mensual del consumo de 18 distribuidoras que venden en Sistema Interconectado Central (SIC). En el estudio se explota la información de corte transversal inherente en un panel, que les permite obtener estimaciones más precisas. Al mismo tiempo la periodicidad mensual de los datos permite estimar un modelo de ajuste parcial en que el corto plazo es un mes. La contribución metodológica del trabajo consiste en estimar paneles dinámicos con datos mensuales y examinar la forma correcta de estimar un modelo con variables dependiente rezagada medida en forma mensual cuando los tamaños de muestra no son lo suficiente grandes estudios realizados para el Perú:

El Comité de Operación Económica del Sistema Interconectado Nacional COES-SEIN (1996) realizó un estudio de demanda de energía eléctrica donde se plantea un modelo lineal de demanda en el que se considera como variables explicativas la población, el PBI y las tarifas promedio de energía eléctrica. Los autores utilizaron el método de mínimos cuadrados ordinarios (MCO) para estimar el modelo, las series 
con los que se realizó el trabajo después de ser analizadas presentaron problemas de estacionariedad posible relación espúrea. De otra parte, se puede comprobar que la estimación en su conjunto posee cierta inestabilidad hacia el final de la muestra. Además, el modelo econométrico presenta un problema de composición en el crecimiento de la demanda eléctrica. Específicamente, se sobreestima el crecimiento de la demanda atribuido a la población. Finalmente, el reducido número de observaciones es insuficiente para obtener propiedades deseables en los estimadores MCO.

El modelo CISEPA-PUCP (1998) presenta una función de demanda de energía eléctrica con elasticidades (Modelo de ajuste instantáneo) y una función en la que asume divergencia entre la demanda y consumo de energía (Modelo de ajuste parcial), los autores realizan estimaciones grupales teniendo en cuenta nueve categorías de usuarios, agregándolas luego para lograr una demanda global, en el planteamiento inicial de su modelo se puede notar que no consideran a la población como variable explicativa de la demanda como si lo hace el modelo COES-SEIN, de sus resultados y conclusiones finales se puede notar que para algunas agrupaciones el precio de la energía eléctrica es poco significativo .

El modelo de demanda presentado por Macroconsult (2001), realiza un modelo de corrección de errores para el estudio presentado originalmente por COESSEIN, esta mejora metodológica ha permitido tener una función de demanda con efectos de corto y largo plazo y un mejor ajuste econométrico para fines de predicción.

Finalmente, el estudio de OSINERG (2004), construye modelos econométricos alternativos para la realización de proyecciones de la demanda eléctrica los cuales permiten mejorar la eficiencia y la bondad predictiva, así como reducir la discrecionalidad del procedimiento actual. Se estiman tres modelos: el primero de series de tiempo, el segundo de corrección de errores y el tercero desagrega la demanda eléctrica en tres componentes (residencial, minero y el resto de la industria).

\section{MATERIAL Y MÉTODOS}

\section{Metodología de panel de datos}

Según Greene (1999), y Baltagi (2002) podemos decir que, el uso de datos que varían tanto a través del tiempo como del espacio nos permiten construir y validar modelos conocidos como de datos en panel (agrupamiento de observaciones en series de tiempo y transversales a la vez). En forma genérica, un modelo de datos en panel puede ser representado por:

$$
\begin{gathered}
Y_{i t}=\alpha_{i}+X_{i t} \cdot \beta+\mu_{i t} \\
i=1, \ldots, N \\
t=0, \ldots, \mathrm{T}
\end{gathered}
$$

Donde $\boldsymbol{Y}_{i t}$ representa a la variable dependiente de la unidad transversal $i$ en el momento $t, \alpha_{i}$ se considera constante a lo largo del tiempo $t$ y específico para la unidad de sección cruzada individual, $\boldsymbol{X}_{i t}$ representa al conjunto de $\boldsymbol{K}$ variables independientes, excluyendo el intercepto, que vienen a ser los regresores del modelo para cada unidad transversal $i$ en el momento $t ; \beta$ mide el efecto marginal de $X_{i t}$ en el momento $t$ para la $i$-ésima unidad y $\mu_{i t}$ representa el término de error del modelo de la unidad transversal $i$ en el momento $t$. El supuesto estandar es que $\beta$ es constante para todo $\boldsymbol{i} \boldsymbol{y} \boldsymbol{t}$.

Un modelo econométrico de datos en panel es más robusto que un modelo convencional, Baltagi (2002) destaca las siguientes ventajas de los datos en panel respecto a los datos transversales ó de series de tiempo:

1. Puesto que los datos relacionan distritos, individuos, empresas, estados, países, etc. a lo largo del tiempo, no existe límite alguno para la heterogeneidad en estas unidades. Las técnicas de estimación de datos en panel pueden tener en cuenta de manera explícita tal heterogeneidad, al permitir la existencia de variables específicas individuales.

2. Al combinar las series de tiempo de las observaciones transversales, los datos en panel proporcionan una mayor cantidad de datos informativos, más variabilidad, menos colinealidad entre variables, más grados de libertad y una mayor eficiencia: 
- Los datos en panel pueden detectar y medir mejor los efectos que sencillamente no pueden ni siquiera observarse en datos puramente transversales ó de series de tiempo.

- Al estudiar la sección transversal repetida de observaciones, los datos en panel resultan más adecuados para estudiar la dinámica de cambio.

- Los datos en panel permiten estudiar modelos de comportamiento más complejos. Por ejemplo, fenómenos como las economías de escala y el cambio tecnológico pueden manipularse mejor con los datos en panel que por los datos puramente seccionales o de series de tiempo.

\section{RESULTADOS}

a) Demanda de electricidad en baja tensión

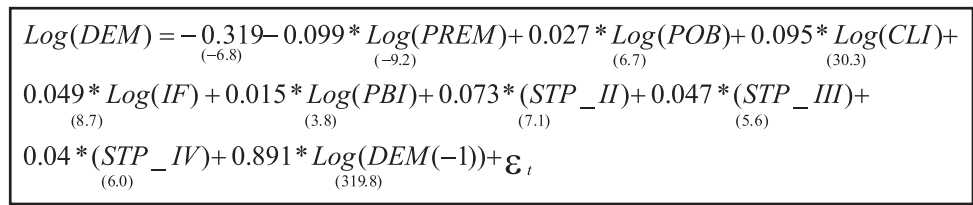

b) Demanda de electricidad en media tensión (Incluye todos los distritos)

$$
\begin{aligned}
& \log (D E M)=-\underset{(-4.99)}{0.3473}-0.1477 * \underset{(-5.37)}{\log }(\text { PREM })+0.0125 * \underset{(4.72)}{\log (C L I)+0.0726} \underset{(6.17)}{\log (I F)+} \\
& 0.1109 * \underset{(9.36)}{\log (S T P}+I I)+0.085 * \underset{(6.86)}{(S T P}-I I I)+0.0307 * \underset{(3.05)}{(S T P}-I V)+0.965 * \underset{(407.02)}{\log (D E M}(-1))+\varepsilon_{t i}
\end{aligned}
$$

c) Demanda de electricidad en media tensión (Excluye los distritos mineros)

$$
\begin{aligned}
& \log (D E M)=-\underset{(-4.5)}{0.303}-0.1565 * \underset{(-5.75)}{\log }(\text { PREM })+0.0078 * \underset{(3.04)}{\log }(C L I)+0.0506 * \underset{(4.44)}{\log }(I F)+ \\
& \left.0.0781 * \underset{(6.96)}{\log }\left(S T P_{-} I I\right)+0.0553 * \underset{(4.82)}{\left(S T P_{-}\right.} I I I\right)+0.0189 * \underset{(1.88)}{\left(S T P_{-} I V\right)+0.9747 * \underset{(488.62)}{\log }(D E M(-1))+\varepsilon_{t i}}
\end{aligned}
$$

d) Elasticidad ( Excluye los distritos mineros en media tensión)

\begin{tabular}{|l|}
\hline BAJA TENSIÓN \\
$\frac{\partial \log (\text { DEM })}{\partial \log (P R E M)}=-0.099$ \\
$\frac{\partial \log (D E M)}{\partial \log (P O B)}=0.027$ \\
$\frac{\partial \log (D E M)}{\partial \log (C L I)}=0.095$ \\
$\frac{\partial \log (D E M)}{\partial \log (I F)}=0.049$ \\
$\frac{\partial \log (D E M)}{\partial \log (P B I)}=0.015$ \\
$\frac{\partial(D E M)}{\partial(\text { STP II })}=0.073$ \\
$\frac{\partial(D E M)}{\partial(S T P-I I I)}=0.047$ \\
$\frac{\partial(D E M)}{\partial(S T P-I V)}=0.04$ \\
$\frac{\partial(D E M)}{\partial(D E M(-1))}=0.891$ \\
\hline
\end{tabular}

\begin{tabular}{|l|}
\hline MEDIA TENSIÓN \\
$\frac{\partial \log (D E M)}{\partial \log (P R E M)}=-0.1565$ \\
$\frac{\partial \log (D E M)}{\partial \log (P O B)}=-----$ \\
$\frac{\partial \log (D E M)}{\partial \log (C L I)}=0.0078$ \\
$\frac{\partial \log (D E M)}{\partial \log (I F)}=0.0506$ \\
$\frac{\partial \log (D E M)}{\partial \log (P B I)}=-----$ \\
$\frac{\partial(D E M)}{\partial(\text { STP II })}=0.0781$ \\
$\frac{\partial(D E M)}{\partial(\text { STP } I I I)}=0.0553$ \\
$\frac{\partial(D E M)}{\partial(\text { STP } I V)}=0.0189$ \\
$\frac{\partial(D E M)}{\partial(D E M(-1))}=0.9747$ \\
\hline
\end{tabular}

En valor absoluto se tiene

\section{BAJA TENSIÓN}

$\eta D E M, P R E M>\eta D E M, C L I>\eta D E M, S T P \quad I I$

\begin{tabular}{l}
\hline MEDIA TENSIÓN \\
$\eta D E M, P R E M \quad>\eta D E M, S T P{ }_{-} I I>\eta D E M, S T P{ }_{-} I I I$ \\
$>\eta D E M, P X_{-} I F+A J$
\end{tabular}


(Excluye los distritos mineros en media tensión)

\begin{tabular}{|l|}
\hline BAJA TENSIÓN \\
$\frac{\partial \log (D E M)}{\partial \log (P R E M)}=-0.099$ \\
$\frac{\partial \log (D E M)}{\partial \log (P O B)}=0.027$ \\
$\frac{\partial \log (D E M)}{\partial \log (C L I)}=0.095$ \\
$\frac{\partial \log (D E M)}{\partial \log (I F)}=0.049$ \\
$\frac{\partial \log (D E M)}{\partial \log (P B I)}=0.015$ \\
$\frac{\partial(D E M)}{\partial\left(S T P \_I I\right)}=0.073$ \\
$\frac{\partial(D E M)}{\partial\left(S T P \_I I\right)}=0.047$ \\
$\frac{\partial(D E M)}{\partial\left(S T P \_I V\right)}=0.04$ \\
$\frac{\partial(D E M)}{\partial(D E M(-1)}=0.891$ \\
\hline
\end{tabular}

\begin{tabular}{|l|}
\hline MEDIA TENSIÓN \\
$\frac{\partial \log (D E M)}{\partial \log (P R E M)}=-0.1477$ \\
$\frac{\partial \log (D E M)}{\partial \log (P O B)}=-----$ \\
$\frac{\partial \log (D E M)}{\partial \log (C L I)}=0.0125$ \\
$\frac{\partial \log (D E M)}{\partial \log (I F)}=0.0726$ \\
$\frac{\partial \log (D E M)}{\partial \log (P B I)}=-----$ \\
$\frac{\partial(D E M)}{\partial\left(S_{P} I I\right)}=0.1109$ \\
$\frac{\partial(D E M)}{\partial\left(S T P \_I I\right)}=0.085$ \\
$\frac{\partial(D E M)}{\partial\left(S T P \_I V\right)}=0.0307$ \\
$\frac{\partial(D E M)}{\partial(D E M(-1)}=0.965$ \\
\hline
\end{tabular}

En valor absoluto se tiene

BAJA TENSIÓN

$\eta D E M, P R E M>\eta D E M, C L I>\eta D E M, S T H$

MEDIA TENSIÓN
$\eta D E M, P R E M>\eta D E M, S T P_{-} I I>\eta D E M, S T$
$>\eta D E M, P X_{-} I F_{-} A J$

\section{e) Pronóstico de la demanda eléctrica en baja tensión}

Pronóstico de Venta de Energía en MT en MWh

a Junio del 2007.

\begin{tabular}{|c|r|}
\hline Fecha & $\begin{array}{c}\text { Demanda_MT } \\
\text { Pronosticada }\end{array}$ \\
\hline 2006_01 & \\
2006_02 & \\
2006_03 & 5759.629 \\
2006_04 & 5914.401 \\
2006_05 & 6119.345 \\
2006_06 & 6248.979 \\
2006_07 & 6435.765 \\
2006_08 & 6546.240 \\
2006_09 & 6467.861 \\
2006_10 & 6602.422 \\
2006_11 & 6488.488 \\
2006_12 & 6609.447 \\
\hline Año 2006 & $\mathbf{7 4 4 2 0 . 3 1 2}$ \\
\hline
\end{tabular}

\begin{tabular}{|r|r|}
\hline Fecha & $\begin{array}{l}\text { Demanda_MT } \\
\text { Pronosticada }\end{array}$ \\
\hline 2007_01 & 6491.710 \\
2007_02 & 6299.790 \\
2007_03 & 6799.655 \\
2007_04 & 6812.572 \\
2007_05 & 7107.423 \\
2007_06 & 7291.062 \\
\hline
\end{tabular}

\section{f) Pronóstico de la demanda eléctrica en media tensión}

Pronóstico de Venta de Energía en BT en MWh al 2010.

\begin{tabular}{|c|c|c|c|}
\hline Fecha & $\begin{array}{l}\text { Demanda_BT } \\
\text { Pronosticada }\end{array}$ & Fecha & $\begin{array}{l}\text { Demanda_BT } \\
\text { Pronosticada }\end{array}$ \\
\hline 2006_01 & & 2007_01 & 27929.302 \\
\hline 2006_02 & & 2007_02 & 26222.650 \\
\hline 2006_03 & 26723.119 & 2007_03 & 28443.441 \\
\hline 2006_04 & 26898.186 & 2007_04 & 28751.819 \\
\hline 2006_05 & 27832.782 & 2007_05 & 29699.380 \\
\hline 2006_06 & 27410.797 & 2007_06 & 29290.299 \\
\hline 2006_07 & 28242.186 & 2007_07 & 30134.532 \\
\hline 2006_08 & 28286.794 & 2007_08 & 30191.924 \\
\hline 2006_09 & 27911.744 & 2007_09 & 29829.601 \\
\hline $2006 \_10$ & 28585.352 & 2007_10 & 30515.877 \\
\hline 2006_11 & 27832.733 & 2007_11 & 29775.869 \\
\hline 2006 & 28635.201 & $2007 \_12$ & 30590.892 \\
\hline Año 2006 & 329175.220 & Año 2007 & 351375.586 \\
\hline Fecha & $\begin{array}{l}\text { Demanda_BT } \\
\text { Pronosticada }\end{array}$ & Fecha & $\begin{array}{l}\text { Demanda_BT } \\
\text { Pronosticada }\end{array}$ \\
\hline 2009_01 & 32011.593 & 2010_01 & 34264.802 \\
\hline 2009_02 & 30329.225 & 2010_02 & 32593.748 \\
\hline 2009_03 & 32574.198 & 2010_03 & 34849.994 \\
\hline 2009_04 & 32906.660 & 2010_04 & 35193.688 \\
\hline 2009_05 & 33878.206 & 2010_05 & 36176.428 \\
\hline 2009_06 & 33493.013 & 2010_06 & 35802.389 \\
\hline 2009_07 & 34361.039 & 2010_07 & 36681.529 \\
\hline 2009_08 & 34442.128 & 2010_08 & 36773.695 \\
\hline 2009_09 & 34103.409 & 2010_09 & 36446.013 \\
\hline 2009_10 & 34813.196 & 2010_10 & 37166.801 \\
\hline 2009_11 & 34096.609 & 2010_11 & 36461.176 \\
\hline 2009_12 & 34934.962 & 2010_12 & 37310.455 \\
\hline Año 2009 & 401944.237 & Año 2010 & 429720.718 \\
\hline
\end{tabular}

Pronóstico de Venta de Energía en MT en MWh al 2010.

\begin{tabular}{|c|c|c|c|c|c|}
\hline Fecha & \begin{tabular}{|l|} 
Demanda_MT \\
Pronosticada \\
\end{tabular} & Fecha & $\begin{array}{l}\text { Demanda_MT } \\
\text { Pronosticada }\end{array}$ & Fecha & $\begin{array}{l}\text { Demanda_MT } \\
\text { Pronosticada }\end{array}$ \\
\hline 2006_01 & & 2007_01 & 5746.892 & \begin{tabular}{|l|} 
2008_01 \\
\end{tabular} & 6409.866 \\
\hline 2006_02 & & 2007_02 & 5517.516 & 2008_02 & 6187.943 \\
\hline 2006_03 & 5341.943 & 2007_03 & 5979.074 & 2008_03 & 6656.763 \\
\hline 2006_04 & 5467.923 & 2007_04 & 952.806 & 2008_04 & 6637.677 \\
\hline 2006_05 & 5643.157 & 2007_05 & 6207.559 & 2008_05 & 6899.450 \\
\hline 2006_06 & 5742.213 & 2007_06 & 6350.149 & 2008_06 & 7048.999 \\
\hline 2006 & 5 & 20 & & 2008_07 & 9.232 \\
\hline 2006_08 & 5975 & 2007_08 & 6599.714 & 2008_08 & 7312.320 \\
\hline 2006_09 & 5864.290 & 2007_09 & 6496.131 & 2008_09 & 7215.570 \\
\hline $2006 \_10$ & 5964.852 & 2007_10 & 6604.616 & 2008_10 & 7330.879 \\
\hline & & & & 2008_11 & 7196.693 \\
\hline $2006-12$ & 5901.240 & $2007-12$ & 6556.631 & $2008-12$ & 7296.462 \\
\hline Año 2006 & 688842.758 & Año 2007 & 74988.233 & Año 2008 & 83411.855 \\
\hline
\end{tabular}

\begin{tabular}{|c|c|c|c|}
\hline Fecha & \begin{tabular}{|l|} 
Demanda_MT \\
Pronosticada
\end{tabular} & Fecha & $\begin{array}{l}\text { Demanda_MT } \\
\text { Pronosticada }\end{array}$ \\
\hline 200901 & 7156.466 & $2010 \quad 01$ & 7987.734 \\
\hline 2009_02 & 6941.379 & 2010_02 & 7779.789 \\
\hline 200903 & 7417.091 & 2010_03 & 8262.607 \\
\hline 2009_04 & 7404.980 & 2010_04 & 8257.568 \\
\hline 2009_05 & 7673.769 & 2010_05 & 8533.388 \\
\hline 2009_06 & 7830.376 & 2010_06 & 8696.994 \\
\hline 200907 & 8007.677 & 2010_07 & 8881.260 \\
\hline 2009_08 & 8107.857 & 2010_08 & 8988.380 \\
\hline 200909 & 8018.213 & 2010_09 & 8905.645 \\
\hline 2009 & 8140.665 & 2010_10 & 9034.978 \\
\hline 2009_11 & 8013.635 & 2010_11 & 8914.792 \\
\hline 2009_12 & 8120.575 & 2010_12 & 9028.544 \\
\hline Año 2009 & 92832.682 & Año 2010 & 103271.680 \\
\hline
\end{tabular}




\section{DISCUSIÓN}

Los modelos estructurales tanto en baja y media tensión mediante la metodología de Panel de datos presentan estacionaridad en las variables, no autocorrelación y no heterocedasticidad. La demanda de electricidad en baja tensión depende principalmente, en orden de importancia, del precio de la electricidad del número de clientes y del sector típico II; mientras que la demanda de electricidad en media tensión, incluyendo los distritos mineros depende del precio del ingreso ajustado y del sector tipico II y III.

El estudio evidencia que la mayor tasa de crecimiento efectivo y proyectado en el mercado de baja tensión se encuentra en la Región Ayacucho, provincia de Huanca Sancos; Región Huancavelica, provincia de Angaraes; Región Huánuco, provincia de Huamalies; Región Junín, provincia de Satipo, y la Región Pasco, provincia de Oxapampa. La mayor tasa de crecimiento efectivo y proyectado en el mercado de media tensión se encuentra en la Región Ayacucho, provincia de Huanta; Región Huancavelica, provincia de Acobamba; Región Huánuco, provincia de Leoncio Prado; Región Junín, provincia de Yauli, y la Región Pasco, provincia de Daniel Alcides Carrión.

La mayor tasa de crecimiento efectivo y proyectado en el mercado regulado de baja tensión a nivel Regional se encuentra en la Región Cusco seguido de la Región Huancavelica y la mayor tasa de crecimiento efectivo y proyectado en el mercado regulado de media tensión a nivel regional se encuentra en la Región Huancavelica y Región Pasco.

\section{f) Pronóstico de la demanda eléctrica total}

La mayor tasa de crecimiento efectivo y proyectado en el mercado regulado de baja tensión a nivel de Unidades de Negocio se encuentra en Unidad de Negocio de Huancavelica, de Tingo María y de Ayacucho.

La mayor tasa de crecimiento efectivo y proyectado en el mercado regulado de media tensión a nivel de Unidades de Negocio se encuentra en la Unidad de Negocio de Pasco, de Tarma y Huancavelica.

A nivel Empresa - mercado de baja tensión- la proyección a corto plazo de la demanda de electricidad para el año 2006 fue de 334,202.6 MWh y para junio del 2007 se espera alcanzar 30,227.1 MWh ; y la proyección a largo plazo de la demanda de electricidad para el año 2006 fue de 329,175.2 MWh y para el año 2010 se espera alcanzar 429,720.7 MWh.

A nivel Empresa - mercado de media tensión- la proyección a corto plazo de la demanda de electricidad para el año 2006 fue de 74,420.3 MWh y para junio del 2007 se espera alcanzar 7,291.0 MWh ; y la proyección a largo plazo de la demanda de electricidad para el año 2006 fue de $68,842.8 \mathrm{MWh}$ y para el año 2010 se espera alcanzar 103,271.7 MWh.

A nivel Empresa la demanda de electricidad total mercado de baja y media tensión- la proyección a corto plazo de la demanda de electricidad para el año 2006 fue de 408,629.950 MWh y para junio del 2007 se espera alcanzar $37,518.220 \mathrm{MWh}$ : y la proyección a largo plazo de la demanda de electricidad para el año 2006 fue de 398,017..978 MWh y para el año 2010 se espera alcanzar 532,992.398 MWh.

\section{LITERATURA CITADA}

Baltagi, B. 2001. Econometric analysis of panel data. Second Edition. John wiley and sons, LTD, p. 230.

Benavente, J. G.; Sanhueza, R. y Serra P. 2004. Estimando la demanda residencial por electricidad en Chile: a doña Juanita le importa el precio. Universidad de Chile, Departamento de Economía.

Chumacero, R. 1996. Estimación de la demanda de energía del sistema interconectado central. Consultoria para la Comisión Nacional de Energía de chile.

CISEPA. 1997. Proyección del consumo mensual de energía eléctrica Junio 1997-Diciembre 2000. Consultoria para la Comisión de Tarifas de Energía. Informe Final.

Comité de Operación Económica del Sistema interconectado Nacional. COES-SEIN. 1996. Documento de trabajo. 
Quantitative Micro Software, LLC, EViews 5.1. 2005. User's Guide., p. 1004.

Greene William H. 2003. Econometric analysis. Fifthy edition. New Jersey, Prentice Hall, p. 1026.

Macroconsul. 2001. Desarrollo de un modelo econométrico de la demanda de energía para el sistema interconectado nacional. Consultoría para la comisión de tarifas de energía. Tomo I y ll.

Macroconsul. 2002. Conversatorio. exposiciones del conversatorio sobre situación económica y política de endeudamiento del Perú. Marzo 2002. Lima.
Mateos, F., M.; Rodríguez Pardina y M. Rossi. 1999. Oferta y demanda de electricidad en la Argentina: un modelo de ecuaciones simultáneas. Centro de estudios macroeconómicos argentinos, Argentina. Centro de Estudios de Regulación, Instituto de Economía -UADE, Argentina.

Martín, A. 1990. Modelo de demanda energética para España. Universidad Autónoma de Madrid, Ciencias Económicas y Empresariales.

Moral, C. J y Vicente. O. J. 2003. Un modelo de Previsión de Demanda Energía Eléctrica. THOR II. Universidad Autónoma de Madrid. 